\title{
Preparation of Silica Nanoparticles via Two-Step Process Utilizing Mixed Chlorosilane Residues
}

\author{
Yonghong Su, Bugang Xu, Jixiang Cai, Liang Chen†, and Bing Huang \\ Faculty of Environmental Science and Engineering, Kunming University of Science and Technology, \\ Kunming, Yunnan 650504, China
}

(Received June 12, 2018; Revised August 21, 2018; Accepted September 4, 2018)

\begin{abstract}
We propose an economic and facile method for the preparation of silica nanoparticles through a two-step process utilizing chlorosilane residues. Mixed chlorosilane residue was alcoholized with absolute ethanol as a first step to form tetraethoxysilane (TEOS). The TEOS was then utilized as a silicon source to synthesize silica nanoparticles in a sol-gel method. The alcoholysis process was designed and optimized utilizing the Taguchi experimental design method and the yield of TEOS was as high as $82.2 \%$ under optimal synthetic conditions. Similarly, the Taguchi method was also utilized to study the effects of synthesis factors on the particle size of silica nanoparticles. The results of statistical analysis indicate that the concentration of ammonia has a greater influence on particle size compared to the mass fractions of TEOS and polyethylene glycol (4.6\% and 9.7\%). The purity of the silica particles synthesized in our experiments is high, but the specific surface area and pore volume are small.
\end{abstract}

Key words : Mixed chlorosilane residues, Taguchi method, Alcoholysis, TEOS, Silica nanoparticles

\section{Introduction}

$\mathrm{W}$ ith the rapid development of the polysilicon industry, a large number of industrial by-product mixed chlorosilane residues are produced in China. Silicon tetrachloride $\left(\mathrm{SiCl}_{4}\right)$ and trichlorosilane $\left(\mathrm{SiHCl}_{3}\right)$ liquids account for up to $80 \%$ of these residues. For each ton of polysilicon produced, the process generates a minimum of approximately 18 tons of $\mathrm{SiCl}_{4}$ liquid waste in some Chinese enterprises, ${ }^{1,2)}$ Mixed chlorosilane residues react violently with water and transform into acids and poisonous hydrogen chloride gas when exposed to humid air, which can not only irritate eyes and respiratory tract systems, but also endanger the environment. ${ }^{3)}$ Currently, hydrolysis is the most common method for treating mixed chlorosilane residues in domestic processes. Although acid hydrolysis is capable of recovering a portion of the hydrogen chloride gas, the silica-containing solid waste produced by hydrolysis is typically landfilled, which results in a significant waste of silica. Therefore, it has become an urgent issue to explore a method for treating mixed chlorosilane residues in accordance with China's Sustainable Development Strategy.

$\mathrm{SiCl}_{4}$ is the most commonly used raw material for the production of silica nanoparticles. The synthesis of silica nanoparticles based on $\mathrm{SiCl}_{4}$ materials is typically achieved through vapor-phase or vapor-liquid reactions. For example, fumed silica nanoparticles are prepared through vapor-

\footnotetext{
Corresponding author : Liang Chen

E-mail : kmchenliang@hotmail.com

Tel : +86-15808857779 Fax : +86-87165173867
}

phase reactions in which $\mathrm{SiCl}_{4}$ is burned in a hydrogen-oxygen flame, but this technology involves high energy consumption, harsh reaction conditions, and large investment. ${ }^{4}$ Yan et al. successfully prepared silica nanoparticles via hydrolysis of $\mathrm{SiCl}_{4}$ vapor with water vapor at low temperature $\left(\sim 150^{\circ} \mathrm{C}\right){ }^{5)}$ Luo et al. reported the production of silica nanoparticles through the hydrolysis of $\mathrm{SiCl}_{4}$ vapor in an alkaline solution. ${ }^{6}$ Hong et al. reported the synthesis of silica nanoparticles through the hydrolysis of $\mathrm{SiCl}_{4}$ vapor in a diffusion flame of air and liquid petrol gas. ${ }^{7}$ In liquid-phase approaches, silica nanoparticles are typically prepared through a precipitation reaction utilizing $\mathrm{SiCl}_{4}$ in a microemulsion system, ${ }^{8}$ but it is difficult to perform such production on a large scale. Xu et al. and Cai et al. synthesized silica particles with particle sizes in the range of tens of microns via hydrolysis of chlorosilane residue in aqueous solutions. ${ }^{910)}$ Overall, many studies on mixed chlorosilane residues as silicon sources utilizing liquid-liquid reactions to prepare silica nanoparticles have been performed, but methods that can be utilized for amplification have rarely been reported.

The sol-gel-based Stöber method is currently the most popularity and widely used method for the synthesis of silica nanoparticles. This method involves the hydrolysis and polymerization of TEOS in a mixed solution of water, solvent, and admixture. Lee et al. prepared silica nanoparticles induced by non-alcoholic solvent addition with TEOS as a precursor. ${ }^{11)}$ Mily et al. prepared silica nanoparticles through a sol-gel process in an alcoholic solution under microwave irradiation in the presence of an ammonium catalyst. ${ }^{12)}$ Based on these studies, one can determine that TEOS is an 
ideal precursor for the preparation of silica nanoparticles.

To the best of our knowledge, $\mathrm{SiCl}_{4}$ and $\mathrm{SiHCl}_{3}$ are the optimal precursors for TEOS preparation, where the relevant reactions are defined by the following equations (1-4):

$$
\begin{array}{ll}
\mathrm{SiCl}_{4}+3 \mathrm{C}_{2} \mathrm{H}_{5} \mathrm{OH} \rightarrow \mathrm{SiCl}\left(\mathrm{OC}_{2} \mathrm{H}_{5}\right)_{3}+\mathrm{HCl} & \text { [Reaction (1)] } \\
\mathrm{SiCl}\left(\mathrm{OC}_{2} \mathrm{H}_{5}\right)_{3}+\mathrm{C}_{2} \mathrm{H}_{5} \mathrm{OH} \leftrightarrow \mathrm{Si}\left(\mathrm{OC}_{2} \mathrm{H}_{5}\right)_{4}+\mathrm{HCl} & \text { [Reaction (2)] } \\
\mathrm{SiHCl}_{3}+3 \mathrm{C}_{2} \mathrm{H}_{5} \mathrm{OH} \rightarrow \mathrm{SiH}\left(\mathrm{OC}_{2} \mathrm{H}_{5}\right)_{3}+3 \mathrm{HCl} & \text { [Reaction (3)] } \\
\mathrm{SiH}\left(\mathrm{OC}_{2} \mathrm{H}_{5}\right)_{3}+\mathrm{C}_{2} \mathrm{H}_{5} \mathrm{OH} \rightarrow \mathrm{Si}\left(\mathrm{OC}_{2} \mathrm{H}_{5}\right)_{4}+\mathrm{H}_{2} & \text { [Reaction (4)] }
\end{array}
$$

A method utilizing mixed chlorosilane residue alcoholized at low temperature as a first step to form TEOS, which is then converted into silica nanoparticles through further hydrolysis and condensation, has not been reported previously. Additionally, there have been no comprehensive studies on the effects of alcoholysis conditions on TEOS yield.

The Taguchi method is a statistical and efficient approach for developing, improving, and optimizing experimental processes. The basic principle of this method is to employ orthogonal arrays to minimize the number of experiments required to analyze the effects of experimental variables on synthetic processes. ${ }^{13)}$ This method can be utilized to determine the optimal conditions for performing a few welldefined experimental sets. In this study, the Taguchi method was utilized to investigate the effects of various reaction parameters on the yield of TEOS, as well as the effects of the synthesis conditions of silica nanoparticles on the final silica particles.

\section{Experimental Procedure}

In this study, the initial solutions were absolute ethanol, ammonium hydroxide, deionized water, hexane, polyethylene glycol (PEG) 1000, calcium oxide, and mixed chlorosilane residues $\left(\mathrm{SiCl}_{4}: 80.67 \mathrm{wt} \%, \mathrm{SiHCl}_{3}: 9.86 \mathrm{wt} \%\right.$, and $\mathrm{HCl}$ : $9.86 \mathrm{wt} \%)$. All materials were utilized without further purification.

\subsection{Experimental design}

Various parameters of the alcoholysis process can affect the yield of TEOS. In this study, experimental design was performed based on to the L9 $\left(3^{4}\right)$ Taguchi orthogonal design process to investigate the influences of various parameters and the optimal conditions for the alcoholysis process. We selected four factors, namely the (1) mass ratio of absolute ethanol to mixed chlorosilane residues, (2) mass ratio of hexane to mixed chlorosilane residues, (3) reaction temperature, and (4) feed rate of absolute ethanol, to study their effects on TEOS yield. For each parameter, we set up three test levels. The four factors and their levels are listed in Table 1. The numbers "1," "2," and " 3 " in Table 1 represent the first, second, and third levels of each factor, respectively. Similarly, the L9 $\left(3^{4}\right)$ Taguchi orthogonal design process for the preparation of nanometer silica from TEOS is detailed in Table 2.

\subsection{Alcoholysis and synthesis}

The alcoholysis reaction was performed in a four-necked flask with a capacity of $500 \mathrm{~mL}$, equipped with a magnetic stirrer, feed pump, and nitrogen supply system. The following summary represents a typical alcoholysis experiment. First, $0.7 \mathrm{M}$ of mixed chlorosilane residues and $0.457 \mathrm{M}$ of hexane were mixed at $20^{\circ} \mathrm{C}$ via magnetic stirring for 10 min. Next, 1.96 M of absolute ethanol was added to the mixture in droplets at a rate of $60 \mathrm{~mL} / \mathrm{min}$ under a nitrogen flow rate of $100 \mathrm{~mL} / \mathrm{min}$. The reaction solution was collected when the reaction was completed and an appropriate amount of calcium oxide was added to remove any remaining hydrochloric acid until the reaction solution became neutral. The calcium oxide was then removed via filtration. Finally, the reaction solution was analyzed via gas chromatography and the TEOS yield was calculated utilizing the external standard method, followed by purification via distillation to extract the TEOS.

Next, hydrolysis and condensation reactions of the TEOS were performed in an alcohol solvent in the presence of

Table 1. Experimental Factors and Levels for Alcoholysis Experiments

\begin{tabular}{lccc}
\hline \multicolumn{1}{c}{ Parameter } & \multicolumn{2}{c}{ Levels } \\
\cline { 2 - 4 } & Low (1) & Medium (2) & High (3) \\
\hline A: Mass ratio of absolute ethanol to mixed chlorosilane residues & $2.8: 1$ & $3: 1$ & $3.2: 1$ \\
B: Temperature $\left[{ }^{\circ} \mathrm{C}\right]$ & 20 & 30 & 40 \\
C: Feed rate $[\mathrm{ml} / \mathrm{min}]$ & 60 & 90 & 120 \\
D: Mass ratio of hexane to mixed chlorosilane residues & $1: 1.53$ & $1.30: 1$ & $1.95: 1$ \\
\hline
\end{tabular}

Table 2. Experimental Factors and Levels for the Synthesis of Silica Particles from TEOS

\begin{tabular}{lccc}
\multicolumn{1}{c}{ Parameter } & \multicolumn{2}{c}{ Levels } \\
\cline { 2 - 4 } & Low (1) & Medium (2) & High (3) \\
\hline E: Molar concentration of TEOS [mol/L] & 0.2 & 0.3 & 0.4 \\
F: Molar concentration of $\mathrm{NH}_{3}[\mathrm{~mol} / \mathrm{L}]$ & 0.2 & 0.3 & 0.4 \\
G: Mass fraction of PEG $1000[\%]$ & 1.69 & 2.11 & 2.53 \\
\hline
\end{tabular}


ammonium hydroxide. In a typical procedure, $150 \mathrm{~mL}$ of absolute ethanol and an appropriate amount of $\mathrm{NH}_{4} \mathrm{OH}$ were blended in a four-necked flask at $25^{\circ} \mathrm{C}$ via magnetic stirring. A known volume of TEOS, absolute ethanol, and PEG 1000 mixture was then added in drops to the above solution. The mixture was then agitated for $48 \mathrm{~h}$ at $25^{\circ} \mathrm{C}$ to obtain white precipitate, which was collected via filtration and washed with deionized water and ethanol several times. Finally, the samples were dried in an oven overnight at $80^{\circ} \mathrm{C}$.

\subsection{Analysis and characterization}

The components of the mixed solution prepared through the alcoholysis reaction were tested and analyzed using a gas chromatography analyzer (Agilent 1790). The yield of TEOS was calculated based on Eq. (5). The compositions of the silica samples were analyzed based on their X-ray fluorescence (XRF, Axios-mAX). The crystal structures of the silica nanoparticles were measured via X-ray diffraction (XRD, EMPYREAN). The morphology of the products was observed via scanning electron microscopy (SEM, Nova NANOSEM-450). The size of the as-synthesized silica particles was measured via dynamic light scattering (MS2000). The surface area and porosity of the products were analyzed by a Quantachrome (USA) device using the adsorption/ desorption isotherms of nitrogen. Thermogravimetric (TG) analysis and differential thermal scanning (DSC) analysis of the sample were performed utilizing a simultaneous thermal analyzer (STA 449F3) with a heating rate of $5^{\circ} \mathrm{C} / \mathrm{min}$ from $30^{\circ} \mathrm{C}$ to $1000^{\circ} \mathrm{C}$ in an atmosphere of argon gas. The surface structures of samples were analyzed via Fourier transform infrared spectrometer (FTIR, NICOLET-IS10).

$$
y=\frac{m_{1} / M_{1}}{m_{2} / M_{2}+m_{3} / M_{3}} \times 100 \%
$$

$y$ : yield of TEOS.

$M_{1}$ : relative molecular mass of TEOS.

$m_{1}$ : mass of TEOS in the reaction mixture.

$m_{2}$ : mass of $\mathrm{SiCl}_{4}$ in chlorosilane residues.

$M_{2}$ : relative molecular mass of $\mathrm{SiCl}_{4}$.

$m_{3}$ : mass of $\mathrm{SiHCl}_{3}$ in chlorosilane residues.

$M_{3}$ : relative molecular mass of $\mathrm{SiHCl}_{3}$.

\section{Results and Discussion}

\subsection{Determination of optimal alcoholysis condi- tions}

To identify the optimal alcoholysis conditions and most important factors affecting the yield of TEOS, the signal-tonoise $(\mathrm{S} / \mathrm{N})$ ratio was utilized in the Taguchi method. The $\mathrm{S} /$ $\mathrm{N}$ ratios are calculated by Eq. (6) when a smaller value is desirable. ${ }^{14)}$

$$
\mathrm{S} / \mathrm{N}=-10 \log \left(\frac{1}{n} \sum_{i=1}^{n} Y_{i}^{2}\right)
$$

In the case where a larger value is desirable, $\mathrm{S} / \mathrm{N}$ ratios are calculated by Eq. (7).

$$
\mathrm{S} / \mathrm{N}=-10 \log \left(\frac{1}{n} \sum_{i=1}^{n} \frac{1}{Y_{i}^{2}}\right)
$$

When a nominal value is desirable, $\mathrm{S} / \mathrm{N}$ ratios are calculated by Eq. (8).

$$
\mathrm{S} / \mathrm{N}=10 \log \left(\frac{\mu^{2}}{\delta^{2}}\right)
$$

where

$$
\begin{aligned}
& \mu^{2}=\frac{1}{n} \sum_{i=1}^{n} Y_{i}^{2} \\
& \delta^{2}=\frac{1}{n-1} \sum_{i=1}^{n}\left(Y_{i}-\mu\right)^{n} .
\end{aligned}
$$

In Eqs. (6)-(10), $n$ is the replication number of the experiment in the orthogonal array and $Y_{i}$ is the measured value of each response. The units of $\mathrm{S} / \mathrm{N}$ ratios are decibels $(\mathrm{dB})$. To identify the optimal conditions for alcoholysis reactions, Eq. (7) (larger value desirable) was utilized to optimize the TEOS yield. Table 3 lists the TEOS yields and $\mathrm{S} / \mathrm{N}$ values for the TEOS obtained in our experiments. Additionally, the mean $\mathrm{S} / \mathrm{N}$ ratio was utilized to estimate the effectiveness of each factor at different levels. For example, the mean $\mathrm{S} / \mathrm{N}$ ratios for the mass ratios of absolute ethanol to mixed chlorosilane residues at levels one, two, and three can be calculated by averaging the $\mathrm{S} / \mathrm{N}$ ratios for experiments $1-3,4-6$, and $7-9$, respectively. The mean $\mathrm{S} / \mathrm{N}$ ratios for each level of the other parameters can be computed in a similar manner (Table 4). The S/N responses for the yields of TEOS are summarized in Table 3.

From Table 4, one can see that the highest maximumminimum value is associated with the feed rate of absolute ethanol, indicating that the most significant influencing factor on the yield of TEOS is the feed rate of absolute ethanol. The order of the influence of each factor on the yield of

Table 3. TEOS Yields and the Corresponding S/N Values Obtained from the Alcoholysis Experiments

\begin{tabular}{ccccccc}
\hline Experiment & A & B & C & D & $\begin{array}{c}\text { TEOS yield } \\
(\%)\end{array}$ & $\begin{array}{c}\text { S/N ratio } \\
(\mathrm{dB})\end{array}$ \\
\hline 1 & 1 & 1 & 1 & 1 & 59.67 & 35.52 \\
2 & 1 & 2 & 2 & 2 & 68.31 & 36.69 \\
3 & 1 & 3 & 3 & 3 & 43.52 & 33.13 \\
4 & 2 & 1 & 2 & 3 & 79.95 & 38.06 \\
5 & 2 & 2 & 3 & 1 & 50.65 & 34.09 \\
6 & 2 & 3 & 1 & 2 & 48.82 & 33.77 \\
7 & 3 & 1 & 3 & 2 & 44.24 & 32.92 \\
8 & 3 & 2 & 1 & 3 & 44.07 & 32.88 \\
9 & 3 & 3 & 2 & 1 & 74.5 & 37.44 \\
\hline
\end{tabular}


Table 4. S/N Response Table for TEOS Yields from the Experiments

\begin{tabular}{|c|c|c|c|c|c|}
\hline \multirow{2}{*}{ Effect } & \multirow{2}{*}{ Parameter } & \multicolumn{4}{|c|}{ Mean $\mathrm{S} / \mathrm{N}$ ratio $(\mathrm{dB})$} \\
\hline & & Level 1 & Level 2 & Level 3 & Maximum-minimum \\
\hline $\mathrm{A}$ & Mass ratio of ethanol to mixed chlorosilane residues & 35.11 & 35.31 & 34.41 & 0.90 \\
\hline $\mathrm{B}$ & Reaction temperature $\left[{ }^{\circ} \mathrm{C}\right]$ & 35.50 & 34.55 & 34.78 & 0.95 \\
\hline $\mathrm{C}$ & Feed rate $[\mathrm{ml} / \mathrm{min}]$ & 34.06 & 37.40 & 33.38 & 4.02 \\
\hline $\mathrm{D}$ & Mass ratio of hexane to mixed chlorosilane residues & 35.68 & 34.46 & 34.69 & 1.22 \\
\hline
\end{tabular}

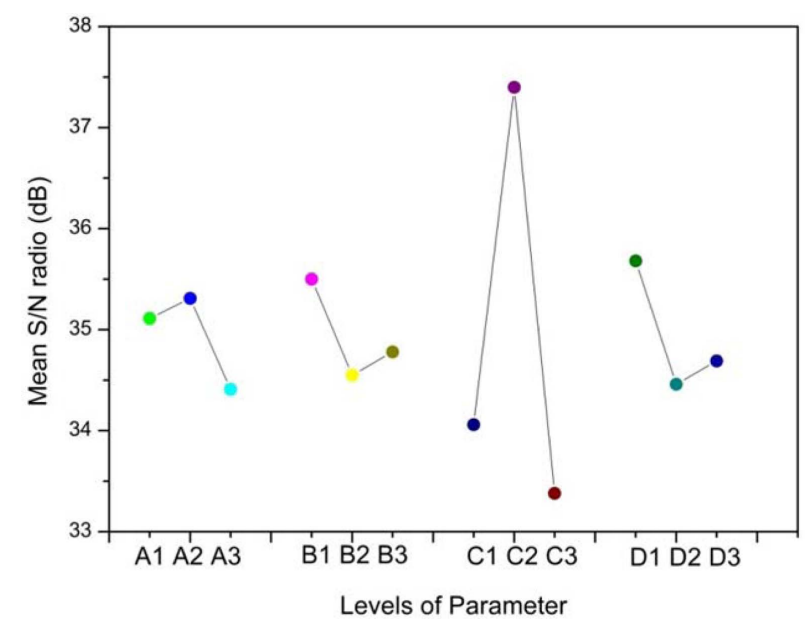

Fig. 1. Mean effect plot for signal-to-noise ratios for TEOS yields.

TEOS is as follows: $\mathrm{C}>\mathrm{D}>\mathrm{B}>\mathrm{A}$. Based on our analysis of $\mathrm{S} / \mathrm{N}$ responses (Fig. 1), the optimal level for each parameter is A2B1C2D1 (B and D at level 1, A and $\mathrm{C}$ at level 2). To estimate the yield of TEOS under these optimal conditions, another experiment was performed. The results revealed that the yield of TEOS reached $82.2 \%$ under optimal conditions.

\subsection{Statistical analysis of the impacts of various factors on silica particle size}

Table 5 lists the mean silica particle sizes and standard deviations calculated in our eight reported experiments. One can see that the mean silica sizes of the samples ranged from 145-381 nm, which proves that the preparation of nanoscale silica is feasible through a two-step process utilizing chlorosilane residue. To quantify the importance of each synthesis factor, analysis of variables (ANOVA) was performed based on the measured mean silica particle sizes. ${ }^{15}$ The analysis results are listed in Table 6 , with headings
Table 5. Mean Silica Particle Sizes Prepared by the Sol-Gel Method

\begin{tabular}{cccccc}
\hline Experiment & E & F & G & $\begin{array}{c}\text { Mean particle size } \\
(\mathrm{nm})\end{array}$ & $\begin{array}{c}\text { SD } \\
( \pm \mathrm{nm})\end{array}$ \\
\hline 1 & 1 & 1 & 1 & 145 & 7.8 \\
2 & 1 & 2 & 2 & 227 & Bimodal \\
3 & 1 & 3 & 3 & 381 & 1.2 \\
4 & 2 & 1 & 2 & 148 & 2.3 \\
5 & 2 & 2 & 3 & 251 & Bimodal \\
6 & 2 & 3 & 1 & 298 & Bimodal \\
7 & 3 & 1 & 3 & 157 & 1.2 \\
8 & 3 & 2 & 1 & 165 & 2.9 \\
9 & 3 & 3 & 2 & 306 & Bimodal \\
\hline
\end{tabular}

representing the sum of squares (SS), degrees of freedom (df), root mean squares (MS), factor of variance-to-error variance ratio $(F)$, and contribution percent $(p)$ of each factor to mean silica size. The results reveal that among the three factors investigated in this study, the molar concentration of $\mathrm{NH}_{3}$ had the greatest impact (85.6\%) on mean silica size, followed by the mass fraction of PEG 1000 (9.7\%) and molar concentration of TEOS (4.6\%). Here, the $\mathrm{pH}$ value mainly affects the sample size by affecting the hydrolysis and condensation rate of TEOS. At high $\mathrm{pH}$ values, the hydrolysis rate is higher than the condensation rate, which leads to the growth of particles dominating nucleation, meaning the synthesized particles are larger. PEG was utilized as a nanostructure control agent because it can adsorb on the surface of particles to exert a steric effect during their preparation, thereby controlling the generation of crystals and suppressing the agglomeration of particles. Finally, small silica particles with good dispersibility were prepared. For the TEOS reactant, under certain conditions, the greater its concentration, the larger the particle size of the synthesized silica particles.

Table 6. Analysis of Variance for Mean Silica Particle Size

\begin{tabular}{cccccc}
\hline Source & SS & df & MS & F-Value & Contribution percent (p)[\%] \\
\hline E & 2613.556 & 2 & 1306.778 & 38.688 & 4.6 \\
F & 48937.556 & 2 & 24468.778 & 724.405 & 85.6 \\
G & 5528.222 & 2 & 2764.111 & 81.832 & 9.7 \\
Residual & 67.556 & 2 & 33.778 & & 0.1 \\
Total & 57146.889 & 2 & & & \\
\hline
\end{tabular}



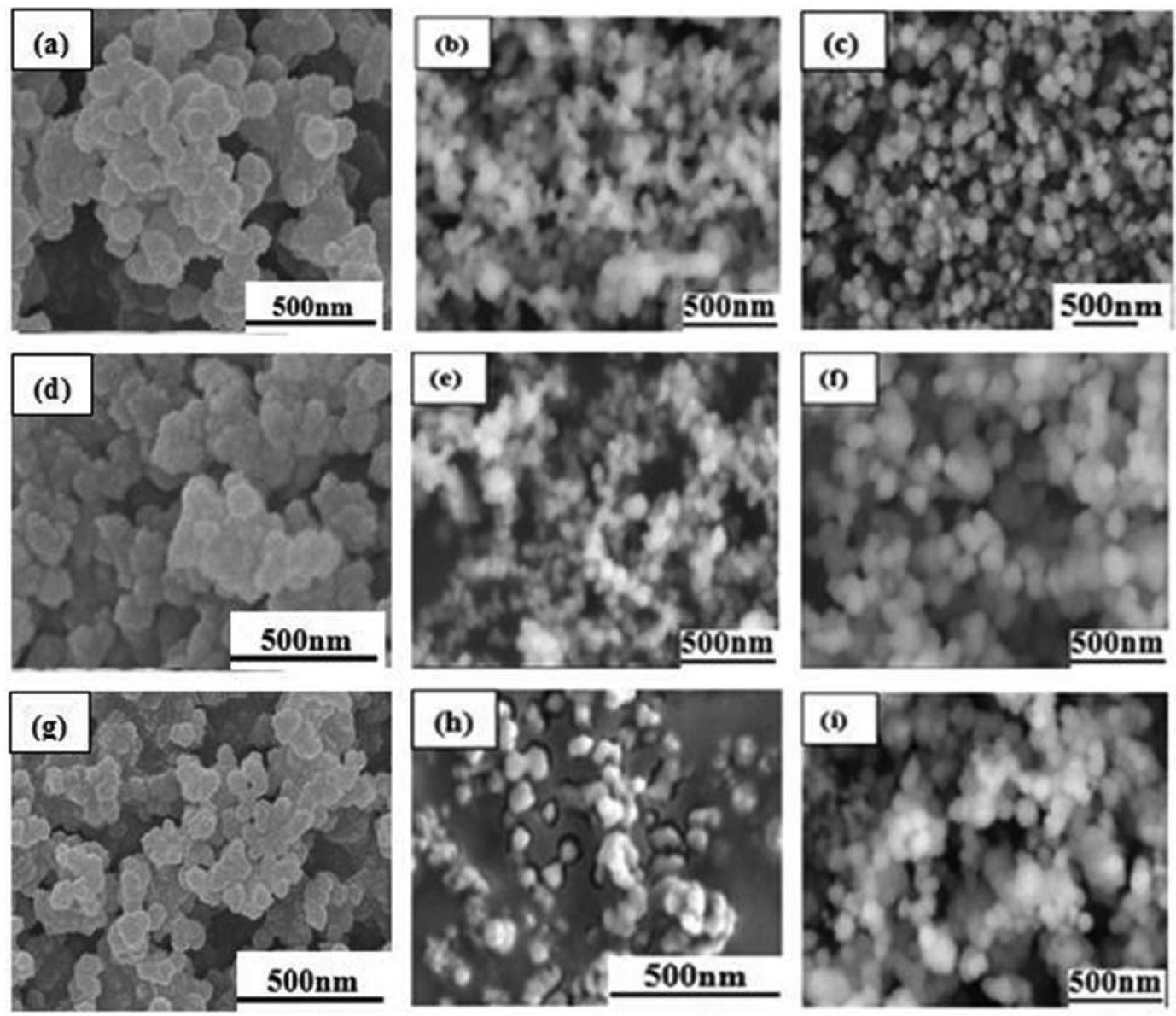

Fig. 2. SEM photographs of synthesized silica nanoparticles designed by the Taguchi method (panels a-i represent samples 1-9, respectively).

\subsection{Characterization of silica particles}

3.3.1. Chemical composition and morphology of samples The chemical compositions of the samples were analyzed based on XRF. Excluding the $\mathrm{H}_{2} \mathrm{O}$ and PEG 1000 contents, the $\mathrm{SiO}_{2}$ content of the samples was greater than or equal to $99.8 \mathrm{wt} \%$. Very few impurities were detected, including $\mathrm{Fe}_{2} \mathrm{O}_{3}, \mathrm{Na}_{2} \mathrm{O}, \mathrm{K}_{2} \mathrm{O}, \mathrm{CaO}, \mathrm{P}_{2} \mathrm{O}_{5}$, and $\mathrm{Cl}$. The content of these impurities was very consistent across different samples. Fig. 2 presents the SEM images of the nine samples synthesized based on the Taguchi experimental design. All samples were composed of small silica particles, but aggregation was significant.

\subsubsection{Structural properties of silica nanoparticles}

To identify the amorphous or crystalline structures of the as-prepared silica powders, powder XRD patterns were utilized. A single broad peak centered at approximately $2 \theta=23^{\circ}$ is shown in Fig. 3, which may be caused by the smaller particle sizes and incomplete inner structures of the spherical nanoparticles. ${ }^{16)}$ This result indicates that the samples were amorphous.

The above analysis reveals that $\mathrm{pH}$ level had the greatest influence on the particle size of the samples. In order to

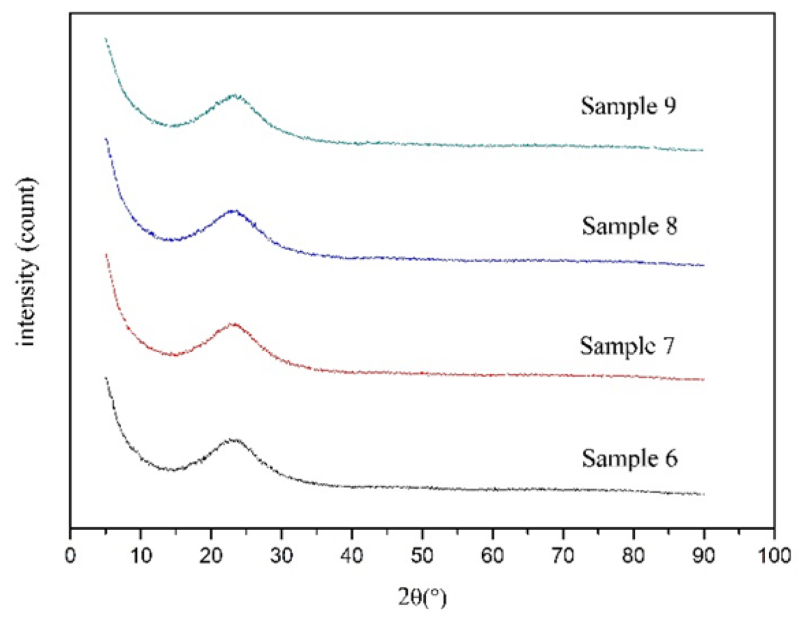

Fig. 3. XRD patterns of samples six, seven, eight, and nine.

understand the characteristics of specific surface area, the pore volumes and pore sizes of the samples synthesized at different $\mathrm{pH}$ values (samples one, two, and three) were investigated via nitrogen adsorption and desorption analysis. The nitrogen adsorption-desorption isotherms and pore distributions of samples one, two, and three are presented 

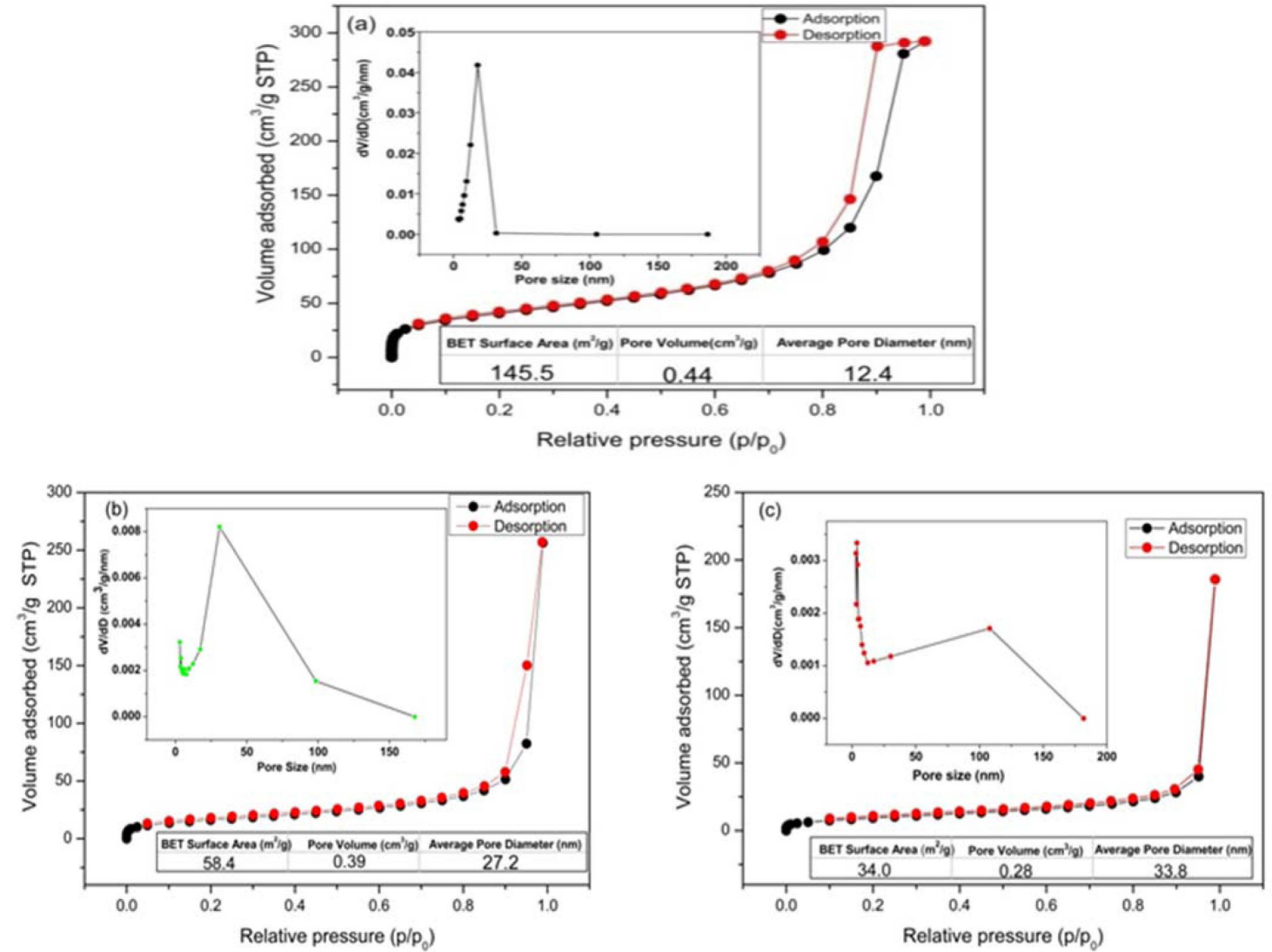

Fig. 4. (a) Nitrogen adsorption-desorption isotherms and pore-size distribution plots for sample one, (b) nitrogen adsorptiondesorption isotherms and pore-size distribution plots for sample two, and (c) nitrogen adsorption-desorption isotherms and pore-size distribution plots for sample three.

in Fig. 4. In this experiment, samples were calcined in air at $550^{\circ} \mathrm{C}$ for $8 \mathrm{~h}$ to ensure complete removal of the PEG 1000 . The nitrogen isotherms for the samples can be divided into two types. Sample one yielded the typical type IV isotherms with $\mathrm{H} 1$ hysteresis loops according to the IUPAC classification. The presence of type $\mathrm{H} 1$ hysteresis loops indicates that sample one is a mesoporous material with relatively small pore diameters. The pore size distribution also supports this conclusion (Fig. 4(a)). Additionally, a sharp peak centered at $17.6 \mathrm{~nm}$ in the pore-size distribution curve was observed.

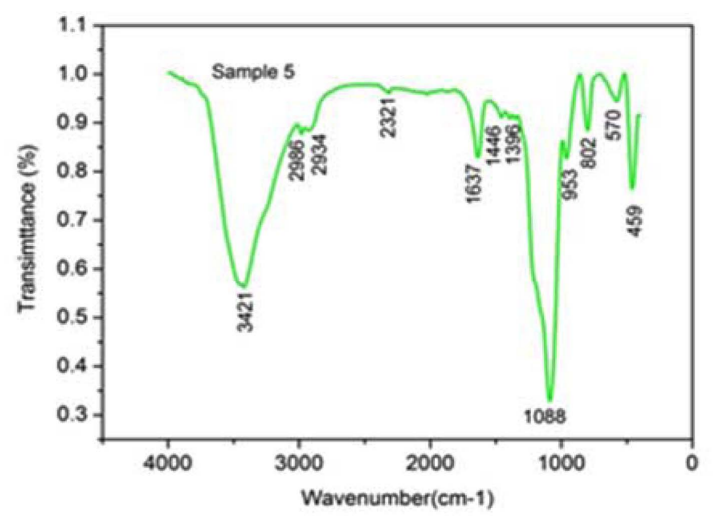

Samples two and three exhibited type IV isotherms with an H3 hysteresis loop, which represents macroporous solids with multilayered adsorption. The macropores likely resulted from the interspaces between small silica nanoparticles, which are also indicated by the hysteresis at high relative pressures. ${ }^{17)}$ A small type of $\mathrm{H} 3$ hysteresis loop at a high relative pressure of approximately 0.8 was observed, which may be a result of the random distribution of pores and interconnecting pore systems. ${ }^{18)}$ The pore size distribution reveals that samples one and two both have relatively wide

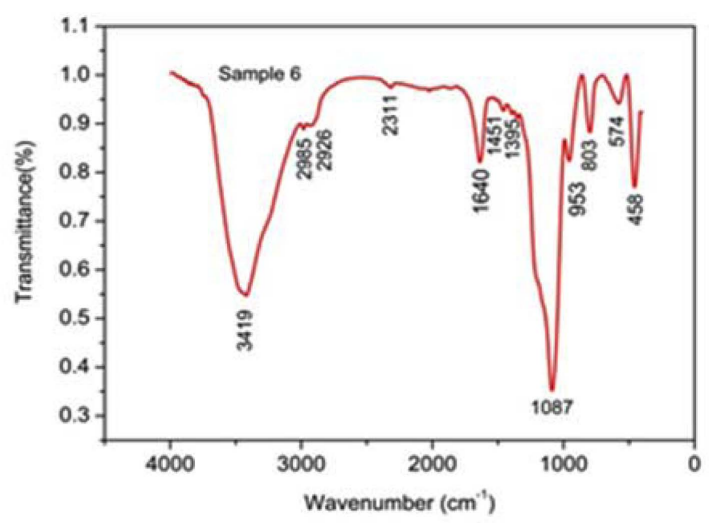

Fig. 5. FT-IR spectra for samples five and six. 
size distributions. The average pore sizes of samples one and two were calculated via the Barrett-Joyner-Halenda method, with results of $27.2 \mathrm{~nm}$ and $33.8 \mathrm{~nm}$, respectively. Samples one, two, and three have low specific surface areas and low pore volumes.

FTIR spectra were utilized to investigate the surface structures of the silica nanoparticles (Fig. 5). The band around 3421 (3419) $\mathrm{cm}^{-1}$ is caused by the stretching vibrations of $\mathrm{OH}$ in adsorbed water molecules. ${ }^{19)}$ Three bands at 459 (458) $\mathrm{cm}^{-1}, 570$ (574) $\mathrm{cm}^{-1}, 802(803) \mathrm{cm}^{-1}$, and 1088 (1087) $\mathrm{cm}^{-1}$ are related to the bending and stretching vibrations of $\mathrm{Si}$ and $\mathrm{O}^{19,20)}$ The peak at $459(458) \mathrm{cm}^{-1}$ cane be attributed to the bending vibrations of Si-O-Si. The band at $802(803) \mathrm{cm}^{-1}$ can be attributed to the symmetric stretching vibrations of Si-O-Si. The very intense and broad peak appearing at $1088(1087) \mathrm{cm}^{-1}$ is related to the asymmetric vibrations of $\mathrm{Si}-\mathrm{O}-\mathrm{Si}$, which reveals that a dense silica network was formed. The band at $1637(1640) \mathrm{cm}^{-1}$ corresponds to $\mathrm{Si}-\mathrm{H}_{2} \mathrm{O}$ flexion. ${ }^{19)}$ The bands at 1396 (1395) $\mathrm{cm}^{-1}, 1446$ (1451) $\mathrm{cm}^{-1}, 2934(2926) \mathrm{cm}^{-1}$, and $2986(2985) \mathrm{cm}^{-1}$ can be attributed to the bending vibrations of $-\mathrm{CH}_{3}$ and $-\mathrm{CH}_{2}$ groups. $^{21,22)}$ Additionally, a band at 2321 (2311) $\mathrm{cm}^{-1}$ was also detected, which corresponds to $\mathrm{Si}-\mathrm{H}$ stretching vibrations.

\subsubsection{Thermal analysis}

The TG and DSC results, as well as their respective thermograms were utilized to evaluate the incidence of physical and chemical changes in the silica nanoparticles in sample three (Fig. 6). The TG curve of the sample reveals three major weight losses. The first weight loss of $2.27 \mathrm{wt} \%$ occurred in the temperature range of $94.0-348.9^{\circ} \mathrm{C}$ and involved the removal of physically combined water. The second weight loss of $15.67 \mathrm{wt} \%$ in the temperature range of $348.9-559^{\circ} \mathrm{C}$ is related to the loss of chemically combined water, pyrolysis of PEG 1000, and silanol group dehydration. The final weight loss of $1.57 \mathrm{wt} \%$ is associated with the dehydration of isolated hydroxyl groups. ${ }^{20)}$ Additionally, the DTS curve reveals that endothermic and exothermic reactions occurred during the heating of the sample. The endothermic peaks at $398.1^{\circ} \mathrm{C}$ correspond to the evaporation of

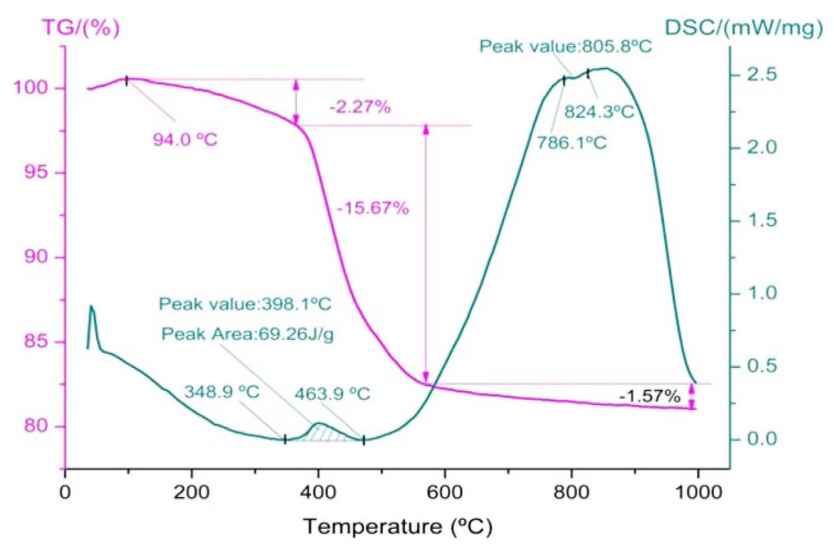

Fig. 6. TG/DSC profiles of sample three. combined water, pyrolysis of PEG 1000 and silanol group dehydration, with a total peak area of $69.26 \mathrm{~J} / \mathrm{g}$. The exothermic peak at $805.8^{\circ} \mathrm{C}$ is associated with phase transition.

\section{Conclusions}

In this paper, a two-step process utilizing chlorosilane residues for the preparation of silica nanoparticles was proposed. This process has great significance for achieving optimal resource utilization. The results of alcoholysis experiments on chlorosilane residues revealed that the feed rate of absolute ethanol is the most significant parameters affecting the yield of TEOS. In descending order of importance, the other parameters affected TEOS yield are the mass ratio of mixed chlorosilane residues to hexane, reaction temperature, and mass ratio of absolute ethanol to mixed chlorosilane residues. The yield of TEOS can reach $82.2 \%$ under optimal alcoholysis conditions.

Silica nanoparticles with mean particle sizes ranging from 145-381 nm were successfully synthesized by controlling the concentrations of TEOS and ammonia, and the mass fraction of PEG 1000. The sequence of parameters influencing particle size was the concentration of ammonia (85.6\%), the mass fraction of PEG 1000 (9.7\%) and concentration of TEOS (4.6\%). XRF and XRD results indicated that the assynthesized silica nanoparticles were amorphous with a purity over $98.8 \%$. SEM results revealed that most of the $\mathrm{SiO}_{2}$ nanoparticles were agglomerated and spherical in shape. Additionally, the synthesized silica created a mesoporous material with low surface area and low pore volume. The proposed two-step process utilizing chlorosilane residues provides a simple and environmentally friendly method for the large-scale production of nanostructured silica materials, which will play a crucial role in the future development of the polysilicon industry.

\section{Acknowledgments}

This research was supported by the National Nature Science Foundation of China (Grant No. 41261079). We are extremely grateful for their contributions.

\section{REFERENCES}

1. J. O. Odden, G. Halvorsen, H. M. Rong, and R. Gløckner, "Comparison of Energy Consumption in Different Production Processes for Solar Grade Silicon," in: The 9th Silicon for the Chemical and Solar Industry, Norway, 2008.

2. C. Wang, T. Wang, P. Li, and Z. Wang, "Recycling of $\mathrm{SiCl}_{4}$ in the Manufacture of Granular Polysilicon in a Fluidized Bed Reactor," Chem. Eng. J., 220 [6] 81-8 (2013).

3. Z. Jianwen, Y. Xinxin, X. Yanan, Z. Jian, Z. Xiaoping, and J. Chunming, "Numerical Investigation on Three-Dimensional Dispersion and Conversion Behaviors of Silicon Tetrachloride Release in the Atmosphere," J. Hazard. Mater., 288 1-16 (2013).

4. G. D. Ulrich, "Theory of Particle Formation and Growth in 
Oxide Synthesis Flames," Combust. Sci. Technol., 4 [1] 47-57 (1971).

5. F. Yan, J. Jiang, X. J. Chen, S. C. Tian, and K. M. Li, "Synthesis and Characterization of Silica Nanoparticles Preparing by Low-Temperature Vapor-Phase Hydrolysis of $\mathrm{SiCl}_{4}$," Ind. Eng. Chem. Res., 53 [30] 11814-90 (2014).

6. Z. Luo, X. Cai, R. Y. Hong, L.S. Wang, and W. G. Feng, "Preparation of Silica Nanoparticles Using Silicon Tetrachloride for Reinforcement of PU," Chem. Eng. J., 187 357-66 (2012).

7. R. R. Hong, B. Feng, Z. Ren, B. Xu, H. Z. Li, Y. Zhang, J. Ding, and D. Wei, "Thermodynamic, Hydrodynamic, Particle Dynamic, and Experimental Analyses of Silica Nanoparticles Synthesis in Diffusion Flame," Can. J. Chem. Eng., 87 [1] 143-56 (2009).

8. X. Zhang and Y. Fan, "Preparation of Spherical Silica Particles in Reverse Micro Emulsions Using Silicon Tetrachloride as Precursor," J. Non-Cryst. Solids, 358 [2] 33741 (2012).

9. B. Xu, L. Chen, Y. Li, P. Luo, and Q. Ma, "Study on the Preparation of Silica Particles from Chlorosilane Residues," J. Non-Cryst. Solids, 463 72-9 (2017).

10. K. D. Kim and H. T. Kim, "Formation of Silica Nanoparticles by Hydrolysis of Teos Using a Mixed SemiBatch/Batch Method," J. Sol-Gel Sci. Technol., 25 [3] 18389 (2002).

11. D. H. Lee, S. W. Han, and D. P. Kang, "Size Change of Silica Nanoparticles Induced by Non-Alcoholic Solvent Addition during Sol-Gel Reaction," J. Sol-Gel Sci. Technol., 74 [1] 78-83 (2015).

12. E. Mily, A. González, J. J. Iruin, L. Irusta, and M. J. Fernández-Berridi, "Silica Nanoparticles Obtained by Microwave Assisted Sol-Gel Process: Multivariate Analysis of the Size and Conversion Dependence," J. Sol-Gel Sci. Technol., 53 [3] 667-72 (2010).

13. M. Edrissi, M. Soleymani, and M. Adinehnia, "Synthesis of Silica Nanoparticles by Ultrasound-Assisted Sol-Gel Method: Optimized by Taguchi Robust Design," Chem. Eng. Technol., 34 [11] 1813-19 (2011).
14. K. D. Kim, D. N. Han, and H. T. Kim, "Optimization of Experimental Conditions Based on the Taguchi Robust Design for the Formation of Nano-Sized Silver Particles by Chemical Reduction Method," Chem. Eng. J., 104 [1] 55-61 (2004).

15. H. Beygi, E. Z. Karimi, R. Farazi, and F. Ebrahimi, "A statistical Approach to Synthesis of Functionally Modified Silica Nanoparticles," J. Alloys Compd., 654 308-14 (2016).

16. T. Gholami, M. Salavati-Niasari, M. Bazarganipour, and E. Noori, "Synthesis and Characterization of Spherical Silica Nanoparticles by Modified Stöber Process Assisted by Organic Ligand," Superlattices Microstruct., 61 33-41 (2013).

17. Y. D. Chiang, H. Y. Lian, S. Y. Leo, S. G. Wang, Y. Yamauchi, and K. C.-W. Wu, "Controlling Particle Size and Structural Properties of Mesoporous Silica Nanoparticles Using the Taguchi Method," J Phys Chem C., 115 [27] 13158-65 (2011).

18. J. C. Groen, L. A. A. Peffer, and J. Pérez-Ramírez, "Pore Size Determination in Modified Micro- and Mesoporous Materials. Pitfalls and Limitations in Gas Adsorption Data Analysis," Microporous Mesoporous Mater., 60 [1-3] 1-17 (2003).

19. N. Pijarn, A. Jaroenworaluck, W. Sunsaneeyametha, and R. Stevens, "Synthesis and Characterization of NanosizedSilica Gels Formed under Controlled Conditions," Powder Technol., 203 [3] 462-68 (2010).

20. J. M. Kim, S. M. Chang, S. M. Kong, K.-S. Kim, J. Kim, and W.-S. Kim, "Control of Hydroxyl Group Content in Silica Particle Synthesized by the Sol-Precipitation Process," Ceram. Int., 35 [3] 1015-19 (2009).

21. Y. D. Wang, C. L. Ma, X. D. Sun, and Heng-de Li, "Synthesis and Characterization of Amorphous $\mathrm{TiO}_{2}$ with Wormhole-like Framework Mesostructure," J. Non-Cryst. Solids, 319 [1-2] 109-16 (2003).

22. L. Zhu, S. Pu, F. Lu, K. Liu, T. Zhu, J. Li, and J. Li, "Preparation of Dispersed Aluminum Hydroxide Nanoparticles via Non-Aqueous Route and Surface Modification," Mater. Chem. Phys., 135 [2-3] 979-84 (2012). 